\title{
Parental COVID-19 vaccine hesitancy for children: vulnerability in an urban hotspot
}

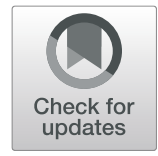

\author{
Nina L. Alfieri ${ }^{1,2,3 \dagger}$, Jennifer D. Kusma ${ }^{1,2,3^{*}+}$, Nia Heard-Garris ${ }^{1,2,3}$, Matthew M. Davis ${ }^{1,2,3,4}$, Emily Golbeck², \\ Leonardo Barrera ${ }^{2}$ and Michelle L. Macy ${ }^{2,3,5}$
}

\begin{abstract}
Objective: To compare hesitancy toward a future COVID-19 vaccine for children of various sociodemographic groups in a major metropolitan area, and to understand how parents obtain information about COVID-19.

Methods: Cross-sectional online survey of parents with children < 18 years old in Chicago and Cook County, Illinois, in June 2020. We used logistic regression to determine the odds of parental COVID-19 vaccine hesitancy (VH) for racial/ethnic and socioeconomic groups, controlling for sociodemographic factors and the sources where parents obtain information regarding COVID-19.

Results: Surveys were received from 1702 parents and 1425 were included in analyses. Overall, 33\% of parents reported VH for their child. COVID-19 VH was higher among non-Hispanic Black parents compared with nonHispanic White parents (Odds Ratio (OR) 2.65, 95\% Confidence Interval (Cl): (1.99-3.53), parents of publicly insured children compared with privately insured (OR 1.93, (1.53-2.42)) and among lower income groups. Parents receive information about COVID-19 from a variety of sources, and those who report using family, internet and health care providers as information sources (compared to those who don't use each respective source) had lower odds of COVID-19 VH for their children.

Conclusions: The highest rates of hesitancy toward a future COVID-19 vaccine were found in demographic groups that have been the most severely affected by the pandemic. These groups may require targeted outreach efforts from trusted sources of information in order to promote equitable uptake of a future COVID-19 vaccine.
\end{abstract}

Keywords: COVID-19, Vaccine hesitancy, Health equity

\section{Background}

The COVID-19 pandemic has resulted in high levels of disease and death tolls $[1,2]$. Of the over 175 million cases worldwide to date as of June 2021, 33.5 million have been in the United States [2] with Black and Hispanic communities experiencing an increased burden of

\footnotetext{
* Correspondence: jkusma@luriechildrens.org

${ }^{+}$Nina L. Alfieri and Jennifer D. Kusma contributed equally as co-first authors. 'Division of Advanced General Pediatrics and Primary Care, Ann \& Robert H. Lurie Children's Hospital of Chicago, 225 E Chicago Ave, Box 162, Chicago, IL 60611, USA

${ }^{2}$ Mary Ann \& J. Milburn Smith Child Health Outcomes, Research, and

Evaluation Center; Stanley Manne Children's Research Institute, Ann \& Robert H. Lurie Children's Hospital of Chicago, Chicago, IL, USA

Full list of author information is available at the end of the article
}

hospitalizations and deaths compared to White counterparts. In the initial wave of the pandemic, urban centers were amongst the hardest-hit areas. Cook County, which includes the city of Chicago, has had over 270,000 cases and over 5000 deaths, as of June 2021 [3], with Black and Hispanic hospitalizations and deaths far surpassing the rates of non-Hispanic Whites respectively $[3,4]$. To mitigate the effects and spread of the COVID-19 pandemic, several important public health measures have been found effective, including universal mask wearing, maintaining physical distances, and limiting social contact. In addition to these measures, two vaccines in the United States have received emergency authorization,

(c) The Author(s). 2021 Open Access This article is licensed under a Creative Commons Attribution 4.0 International License, which permits use, sharing, adaptation, distribution and reproduction in any medium or format, as long as you give appropriate credit to the original author(s) and the source, provide a link to the Creative Commons licence, and indicate if changes were made. The images or other third party material in this article are included in the article's Creative Commons licence, unless indicated otherwise in a credit line to the material. If material is not included in the article's Creative Commons licence and your intended use is not permitted by statutory regulation or exceeds the permitted use, you will need to obtain permission directly from the copyright holder. To view a copy of this licence, visit http://creativecommons.org/licenses/by/4.0/ The Creative Commons Public Domain Dedication waiver (http://creativecommons.org/publicdomain/zero/1.0/) applies to the data made available in this article, unless otherwise stated in a credit line to the data. 
with one approved for individuals as young as 12 years old as of June 2021 . When the vaccine initially received emergency authorization, vaccines were being administered to priority groups such as health care professionals, essential workers, and individuals over the age of 65 , with guidelines subsequently expanding [5]. Vaccine data from clinical trials has shown promise in preventing recipients from morbidity and mortality due to COVID19, and there is hope for decreasing viral transmission and therefore disease volume and burden [5].

In order for a vaccine to be effective in controlling the spread of COVID-19 it is estimated that $67 \%$ of the population will need to receive the vaccine to reach herd immunity [6]. Recent data suggests that countries like the United States may never achieve herd immunity, but as children make up $22 \%$ of the American population, including children in vaccination efforts and plans is imperative for increasing community protection against COVID-19 [7, 8]. With vaccination efforts underway for some adults, it is important to recognize that many adults themselves may decline or defer the vaccine. Unfortunately, vaccine hesitancy, skepticism, and refusal have been a longstanding public health problem for routine immunizations and the annual influenza vaccine for both children and adults. Lower vaccine confidence and uptake has been observed in certain sociodemographic groups including racial/ethnic minorities $[9,10]$ and those with lower socioeconomic status [11].

Understanding the differences in COVID-19 vaccine hesitancy across varying communities and sociodemographic groups is critical for the identification of populations for whom the currently available COVID-19 vaccine information may be insufficient to promote uptake. With this knowledge, vaccine communication and distribution strategies could be tailored to hesitant groups. The objectives of this study were twofold: 1) to compare COVID-19 vaccine hesitancy in sociodemographic groups with higher rates of COVID-19 versus less affected groups, and, 2) to understand how members of these groups receive information about COVID-19 during the first wave of the pandemic.

\section{Methods}

This study consisted of a cross-sectional online survey of parents with children $<18$ years who live in the Chicago metropolitan area and the surrounding suburbs that make up Cook County, IL, as the area was beginning to reopen after the first wave of COVID-19 in June 2020.

\section{Setting and participants}

We used the Qualtrics ${ }^{\mathrm{XM}}$ online survey platform to distribute our survey to parents representative of the racial and ethnic composition of Cook County based on 2010
US Census data. This was done purposefully, using panels of existing survey participants.

\section{Survey instrument development}

The study team developed unique survey questions and also incorporated items from previously published surveys regarding parents and children's responses to epidemics, disasters such as hurricanes, and pandemics including Zika virus [12-15]. We pre-tested the survey with 27 respondents on the Qualtrics ${ }^{\mathrm{XM}}$ platform to ensure proper survey functionality and clarity.

\section{Measures}

\section{Sociodemographic measures}

Our survey assessed demographic characteristics including respondent race and ethnicity, family income, and child insurance type. Race and ethnicity information was collected by asking respondents "what is your race?" and "what is your ethnicity" as two distinct questions. From these responses, a composite race/ethnicity variable was created: non-Hispanic White, non-Hispanic Black, Hispanic, and non-Hispanic mixed-race/other. We asked respondents to estimate their annual household income in categories based on federal poverty levels including: "< $\$ 39,999 ”, \quad$ \$40,000-\$79,999”, “\$80,000-\$149,999”, “> $\$ 150,000$ ", "I'm not sure", or "prefer not to answer". We collapsed no answer, I'm not sure, and prefer not to answer into one category we labeled "did not disclose". Insurance type was identified by asking if their child had private, public, or no insurance.

\section{Sources of COVID-19 information measure}

We asked respondents to select all that apply from an 11-item list to indicate how they received information about COVID-19. Response options included: family/ friends/word of mouth, internet, social media, Department of Health/Centers for Disease Control (CDC)/Government Agency ("Government Agencies"), newspaper (print or online), TV, radio, podcasts, church/place of worship, health care provider, or other. In addition, we asked parents to indicate their level of confidence as "a lot", "some", or "little to no" in each information source they reported using. These two items (use of each source of information and confidence in each source of information) were combined into a new dichotomous variable that included "using" the source of information (having reported "a lot" or "some" confidence) versus "not using" the source (reporting "little to no" confidence or not using the source of information). This dichotomous variable was used in our logistic regression models.

\section{COVID-19 vaccine hesitancy measure}

To understand willingness to obtain a future COVID-19 vaccine, respondents were asked "If a new vaccine 
against COVID-19 became available, how likely would you be to get your child vaccinated?" Respondent parents answered for their child, with responses of "very likely", "somewhat likely", "not likely", and "I'm not sure". We dichotomized responses as having vaccine hesitancy (VH) for those who responded "not likely" or "not sure" and vaccine amenable for those who responded they were "very likely" or "somewhat likely" to get a future COVID-19 vaccine.

\section{Survey distribution}

Recruitment occurred using Qualtrics ${ }^{\mathrm{XM}}$ "dynamic surveys" and their online dashboard-style portal on which surveys are distributed to eligible panel members who $\log$ onto the portal and access surveys as they become available. The survey was made available in English and Spanish. Survey responses were collected from June 8, 2020 to June 29, 2020. To enhance response rates, Qualtrics ${ }^{\mathrm{XM}}$ utilized an automated reminder option based on respondents' preference.

To ensure data quality, the following checks were put into place [1] participants' surveys were terminated if they were found to be "speeding" [16] as indicated by one-half the median of the completion speed during the pilot [2], only unique IP address were included to prevent duplicate responses, and [3] surveys were excluded if respondents chose the same answer for every question, also known as "straight lining" [16].

\section{Ethical considerations}

The study was considered exempt human subjects research by the Ann \& Robert H Lurie Children's Hospital of Chicago Institutional Review Board. Incentives for participation were determined by Qualtrics ${ }^{\mathrm{XM}}$ based on time spent completing the survey, and compensation on similar surveys, which Qualtrics ${ }^{\mathrm{XM}}$ estimated to be about $\$ 5$ per survey.

\section{Analysis}

Our dichotomous outcome of interest was vaccine hesitancy (VH). Predictors of interest were race/ethnicity, income, insurance type, and sources of information. First, bivariate associations between $\mathrm{VH}$ and our predictors of interest were tested. Next we built a multivariate logistic regression model of COVID-19 VH, controlling for parent race/ethnicity, annual household income, child insurance type, and sources of information in which parents reported confidence. All covariates that were significant $(p<0.05)$ in the multiple variable model were retained. The sources of information that did not retain significance were dropped from the final model. A goodness-of-fit test for the adjusted logistic regression model was performed using the Hosmer-Lemeshow test, and was significant with a $p$ value of 0.09 .
Factor analysis and Cronbach alpha testing were performed to determine if we could group the sources of information into clusters. There was no significant grouping of the sources of information (results not shown), so sources were analyzed individually. Statistical analysis was performed in Stata 15 (Stata Corp., College Station, TX).

\section{Results}

Within our target demographic, 5472 panelists who were offered the survey opened it. Of those that opened the survey 2100 started the survey and 1702 respondents completed it in entirety, for an overall response rate of $38.4 \%$ and a survey completion rate of $81.0 \%$. We excluded 277 responses $(16 \%)$ due to quality control concerns, for an overall sample size of 1425 quality responses. In Table 1 we show characteristics of our survey participants. Survey respondents reflected that of Cook County census data with $40 \%$ of participants identifying as non-Hispanic

Table 1 Study demographics

\begin{tabular}{|c|c|c|}
\hline & $\begin{array}{l}\text { Frequency } \\
(N=1425)\end{array}$ & Percentage (\%) \\
\hline \multicolumn{3}{|l|}{ Race/Ethnicity } \\
\hline Non-Hispanic White & 564 & 40 \\
\hline Hispanic (Any Race) & 391 & 27 \\
\hline Non-Hispanic Black & 338 & 24 \\
\hline Non-Hispanic Multi-race/Other & 132 & 9 \\
\hline \multicolumn{3}{|l|}{ Insurance Type (March 1st) } \\
\hline Private & 748 & 53 \\
\hline Public & 596 & 42 \\
\hline None & 81 & 6 \\
\hline \multicolumn{3}{|l|}{ Income (USD) } \\
\hline$<\$ 39,999$ & 366 & 26 \\
\hline$\$ 40 k-\$ 79,999$ & 408 & 29 \\
\hline$\$ 80 k-\$ 149,999$ & 357 & 25 \\
\hline$>\$ 150 k$ & 219 & 15 \\
\hline Did not disclose & 75 & 5 \\
\hline \multicolumn{3}{|l|}{ Source of Information } \\
\hline Internet & 890 & 63 \\
\hline TV & 853 & 60 \\
\hline Social Media & 563 & 40 \\
\hline Government Agency & 556 & 39 \\
\hline Family/Friends/Word of Mouth & 536 & 38 \\
\hline Newspaper & 367 & 26 \\
\hline Health Care Provider & 337 & 24 \\
\hline Radio & 319 & 22 \\
\hline Podcast & 123 & 9 \\
\hline Church/Place of Worship & 63 & 4 \\
\hline
\end{tabular}


White, 24\% non-Hispanic Black, 27\% Hispanic and 9\% non-Hispanic multi-race/other. Twenty-nine parents completed the Spanish language version of the survey. The majority of our survey respondents' child [ren] had health insurance (91\%). Of those, 53\% had private and $42 \%$ had public health insurance. Across annual household incomes $26 \%$ reported < \$39,999, 29\% reported $\$ 40,000-\$ 79,999,25 \%$ reported $\$ 80,000$ - $\$ 149,999,15 \%$ reported $>\$ 150,000$ and $5 \%$ did not disclose.

\section{Hesitancy toward a future COVID-19 vaccine}

Overall, 33\% of parents reported COVID-19 VH for their child. Figure 1 shows respondents' hesitancy, broken down into those "not sure" and those "not likely" to receive a future COVID-19 vaccine for their child across demographic characteristics. Differences in COVID-19 VH were observed by racial/ethnic groups. Forty-eight percent of non-Hispanic Black parents, 33\% of Hispanic parents, and $26 \%$ of non-Hispanic White parents were hesitant about their child receiving the COVID-19 vaccine. Of parents whose children had private health insurance 26\% reported COVID-19 VH for their child. Of parents whose children had public health insurance, $41 \%$ reported COVID-19 VH for their child. Of the parents whose children had no insurance, $43 \%$ reported COVID-19 VH for their child ( $p<0.001$ for all health insurance comparisons). Parents who had an annual household income > \$150,000 reported less COVID-19 VH than parents who had an annual household income of less than $\$ 40,000$ (7\% vs. $44 \% \mathrm{VH}$ for children, respectively, $p<0.001$ ).

\section{Sources of information}

Of the 11 information source options (Fig. 2), the majority of respondents (67\%) used the internet as a source of information, with only $26 \%$ of those reporting having confidence in that source of information. The greatest level of confidence was in government agencies, for which $52 \%$ of respondents reported using as a source of information and $48 \%$ of respondents having confidence in the source. This was followed by health care providers (used by $40 \%$ ) with $44 \%$ of people having confidence in the source. Very few respondents selected "other", 33 respondents (2\%), and this was dropped from subsequent analyses. All information sources in which parents were confident were found to be significantly associated with lower odds of vaccine hesitancy, except for religious sources $(p<0.001)$ (Table 2$)$.

\section{Associations with vaccine hesitancy for child}

Non-Hispanic Black respondents had significantly higher odds (Odds Ratio $(\mathrm{OR})=2.65, p<0.001$ ) of having COVID-19 VH for their child [ren] as compared to nonHispanic white counterparts, and this relationship was maintained when adjusting for income, insurance type, and source of information (Table 2). Families with lower income had greater odds of COVID-19 VH than families in the highest income bracket. There was no interaction

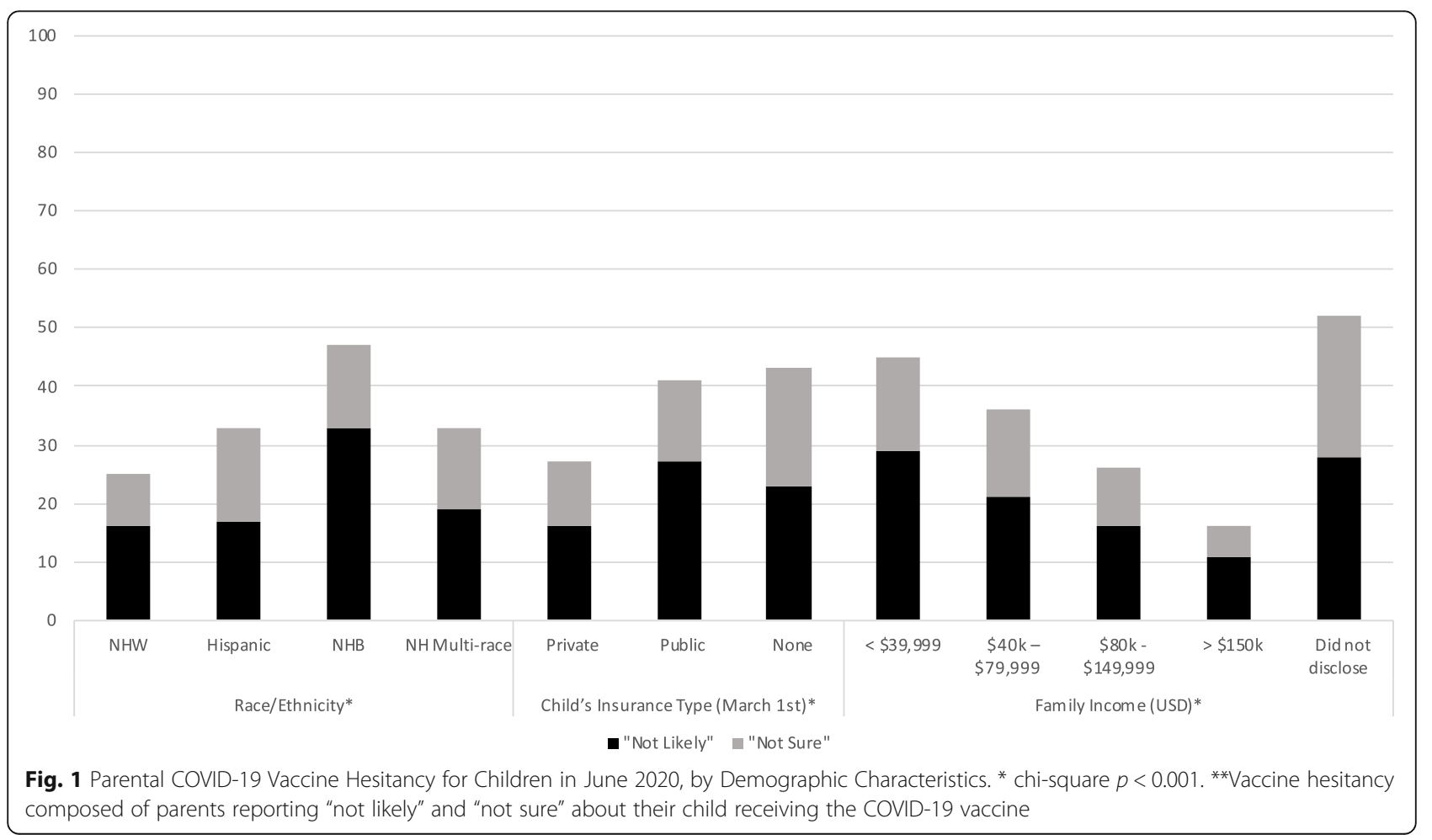




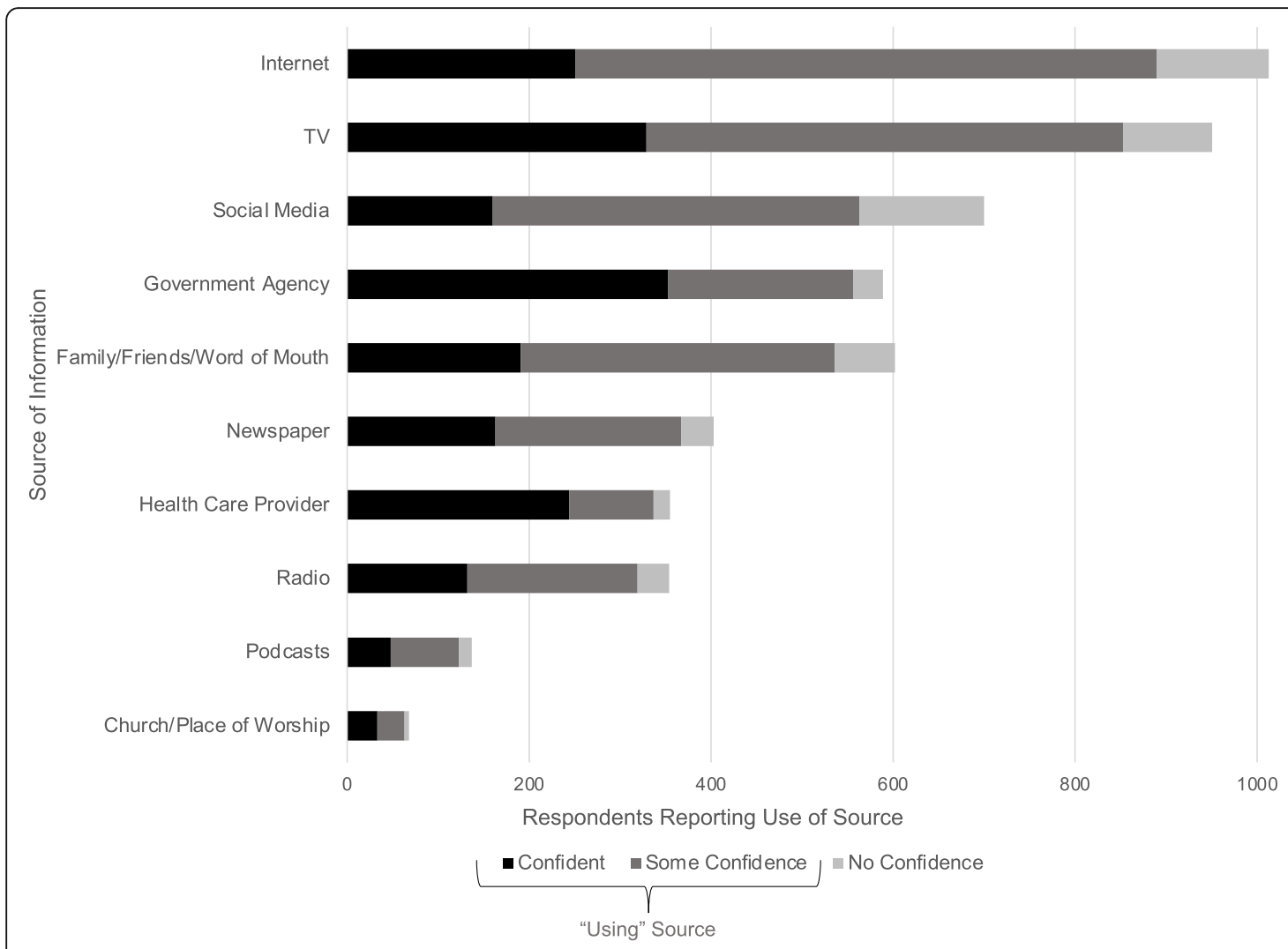

Fig. 2 Sources of Information Regarding COVID-19 Used by Parents ( $n=1425)$

found between race/ethnicity and income (result not shown). Respondents with public insurance had higher odds (OR 1.9, $p<0.001$ ) of being hesitant about having their child receive a future COVID-19 vaccine compared to those with private insurance - which persisted after adjusting. Finally, those who used family, internet and health care providers as information sources had lower odds of COVID-19 VH for their child $(p<0.001)$ when compared to those who did not use each source respectively. For example, parents who used family as a source of information regarding the COVID-19 pandemic had lower odds of COVID-19 VH for their child when compared to parents who did not use family as a source of information.

\section{Discussion}

This study helps to better identify and elucidate parental COVID-19 vaccine hesitancy for children, and investigated associations between COVID-19 VH and sociodemographic characteristics including sources of COVID19 information. Non-Hispanic Black parents, parents with lower income, and parents of children with public health insurance had higher odds of $\mathrm{VH}$ than comparison peers. Additionally, we found lower odds of $\mathrm{VH}$ in parents who used family, internet, and health care providers as sources of information about COVID-19 compared to parents who did not use these sources. Identifying which groups may be most hesitant, and which informational sources may be leveraged, are together important to disseminate accurate and persuasive information regarding a future COVID-19 vaccine to vulnerable groups. As vaccines are becoming available with emergency authorization from the FDA, and with continued spikes in COVID-19 cases, it is of the utmost importance that we are able to widely distribute the vaccine, with emphasis on groups at high, to protect all people.

In our study we found that one third of parents have COVID-19 VH for their child. Hesitancy toward a future COVID-19 vaccine is much higher than for other vaccines. Recent data shows that $6.1 \%$ of U.S. parents are hesitant about routine vaccines and $25.8 \%$ of parents are hesitant about the annual influenza vaccine [11]. There will likely be similar barriers to uptake of a COVID-19 vaccine, highlighting the importance of anticipating and responding to vaccine hesitancy during the early planning stages of COVID-19 vaccine distribution strategies [11]. A CDC strategy already underway is the "Vaccinate with Confidence" framework, which empowers healthcare professionals in reinforcing the safety and importance of vaccinations [17]. The principles of this program can be applied to routine vaccines, the annual influenza vaccine, 
Table 2 Associations of Vaccine Hesitancy for Children

\begin{tabular}{|c|c|c|}
\hline & Child Vaccine Hesitancy OR $(95 \% \mathrm{Cl})$ & $\begin{array}{l}\text { Child Vaccine Hesitancy aOR } \\
(95 \% \mathrm{Cl})\end{array}$ \\
\hline \multicolumn{3}{|l|}{ Race/Ethnicity } \\
\hline Non-Hispanic White & Reference & Reference \\
\hline Hispanic & $1.44^{*}(1.08-1.91)$ & $0.98(0.72-1.34)$ \\
\hline Non-Hispanic Black & $2.65^{* * *}(1.99-3.53)$ & $1.75^{* * *}(1.28-2.39)$ \\
\hline Multiracial/Other & $1.41(0.93-2.12)$ & $1.08(0.70-1.67)$ \\
\hline \multicolumn{3}{|l|}{ Income (USD) } \\
\hline$>\$ 150 k$ & Reference & Reference \\
\hline$\$ 80 k-\$ 149,999$ & $1.80^{* *}(1.17-2.77)$ & $1.70 * *(1.09-2.64)$ \\
\hline$\$ 40 k-\$ 79,999$ & $3.06^{* * *}(2.02-4.62)$ & $2.59 * * *(1.68-3.99)$ \\
\hline$<\$ 39,999$ & $4.17^{* * *}(2.75-6.33)$ & $2.86^{* * *}(1.80-4.53)$ \\
\hline Did not disclose & $5.70^{* * *}(3.19-10.17)$ & $4.27^{* * *}(2.30-7.92)$ \\
\hline \multicolumn{3}{|l|}{ Insurance Type } \\
\hline Private & Reference & Reference \\
\hline Public & $1.93^{* * *}(1.53-2.42)$ & $1.33^{*}(1.01-1.75)$ \\
\hline None & $2.11^{* *}(1.32-3.38)$ & $1.13(0.67-1.91)$ \\
\hline \multicolumn{3}{|l|}{ Source of Information } \\
\hline Internet & $0.54^{* * *}(0.43-0.68)$ & $0.58^{* * *}(0.46-0.74)$ \\
\hline TV & $0.60^{* * *}(0.48-0.75)$ & - \\
\hline Social Media & $0.67^{* *}(0.54-0.85)$ & - \\
\hline Government Agency & $0.66^{* * *}(0.52-0.83)$ & - \\
\hline Family/Friends/Word of Mouth & $0.58^{* * *}(0.46-0.73)$ & $0.69^{* *}(0.53-0.88)$ \\
\hline Newspaper & $0.54^{* * *}(0.41-0.71)$ & - \\
\hline Health Care Provider & $0.56^{* * *}(0.43-0.74)$ & $0.64^{* *}(0.48-0.86)$ \\
\hline Radio & $0.62^{* *}(0.47-0.82)$ & - \\
\hline Podcast & $0.48^{* *}(0.30-0.76)$ & - \\
\hline Religion & $0.78(0.45-1.37)$ & - \\
\hline
\end{tabular}

and now the COVID-19 vaccine. In this study, we identified differences in COVID-19 VH by demographic characteristics. Vaccine hesitancy is a deadly problem, noted by the World Health Organization as a leading threat to global health in regard to known vaccine preventable illnesses [18]. While vaccine hesitancy is complex, it is imperative to explore and address specific concerns for optimal counseling and vaccine uptake [19].

Our finding of greater $\mathrm{VH}$ among non-Hispanic Black parents is consistent with prior research indicating that Black parents have higher hesitancy about vaccination $[11,20]$. These findings are important given the disproportionate negative impact of the COVID-19 pandemic on Black communities in Chicago and throughout the United States [21, 22]. Therefore, it is of great concern that hesitancy toward a potential COVID-19 vaccine may further render Black families more vulnerable to COVID-19. Minority populations were purposefully sampled and included in COVID vaccine trials, and it is of the utmost importance to ensure the trial results are clearly communicated and the safety data is easily accessible to the public [23]. Clear communication that shares vaccine safety and efficacy data and promotes vaccination will enhance uptake of the COVID vaccine across diverse populations, as the vaccine becomes more widely available. It is crucial that further studies investigate and address reasons behind the potential hesitancy toward a COVID-19 vaccine in communities of color. Mistrust in the government and in research may be a key factor in COVID-19 VH. A study by Quinn et al. found that Black adults report hesitancy about vaccines, specifically regarding the motives of governmental agencies not "really caring about us [Black Americans]" or researchers using Black communities as "guinea pigs", also citing the Tuskegee Syphilis experiments as a source of mistrust [24]. However, in addition to mistrust in medicine and research, other factors leading to vaccine hesitancy in Black Americans merits further study. 
It is also important to note that our study showed higher vaccine hesitancy in families with lower household income, independent of race/ethnicity, which is aligned with other recent studies of vaccine hesitancy [25]. Plans for vaccine distribution and vaccine education will need to consider COVID-19 VH in groups that have been historically marginalized, to work towards equitable access to any potential COVID-19 vaccine. Further, our results should compel public health departments and community health organizations to develop effective and culturallysensitive approaches to promote equal uptake of COVID19 vaccine across all populations.

In addition to identifying demographic groups that may be hesitant about a COVID-19 vaccine we also characterized the association between information sources and COVID-19 VH. We found that most of the survey respondents use the internet as a source of information about COVID-19 and they reported lower COVID-19 VH. Previous randomized control trials of interventions targeting vaccine hesitancy suggested that web-based information can help improve vaccination rates, and lessen parental hesitancy towards vaccines [26, 27]. Our findings also show that using family, internet and health care providers as sources of information about COVID-19, was associated with significantly lower odds of having COVID-19 VH when compared to those not using those sources. Vaccine education interventions that use multi-modal formats (e.g., internet and healthcare providers) and are consumed by family and friends within communities may help curb COVID-19 vaccine hesitancy as we strive for herd immunity. Strategies that our participants report using are already being leveraged, including broadcasted vaccinations of public officials against COVID-19, positive vaccine messaging on social media and the internet, and public information campaigns to increase public access to scientific evidence.

Our study should be interpreted within the context of limitations, including survey validation, potential generalizability, and timing. First, although our vaccine hesitancy item was modeled after validated surveys, our survey is not validated due to the novelty of the topic. In addition, our study examines potential attitudes toward a COVID-19 vaccine in June 2020, before a safe and effective vaccine had been created and tested, or disseminated. As such, the information we have collected reflects parental intentions to vaccinate their child as of June 2020 and may not be reflective of actual decisions surrounding vaccination when their child becomes eligible for a COVID-19 vaccine. The information gained from our study can be used to inform public health initiatives to address concerns and hesitancy from parents about the COVID-19 vaccine. Future work to examine changes in parental hesitancy towards a COVID-19 vaccine over time can help to understand changing intent as COVID-19 vaccines become available to children across different age groups. Although we achieved demographic diversity in our study population, mirroring Cook County, our findings may not be generalizable to more rural areas or areas that had lower rates of COVID-19 infections. While we recognize the benefit of national samples, there are also specific benefits to representative household samples in large urban areas in which research questions can be examined with greater granular detail about the local socio-econo-environmental context for each respondent, and where samples also reflect greater demographic diversity than in typical national samples. In the case of our study, we believe that findings from our sample of households in Chicago (the 3rd largest urban area in the United States), for which we had local details about COVID case burden, are likely to be very applicable across other metropolitan areas in the nation where the burden of COVID illness and mortality has been concentrated during the pandemic. Our results may also be limited by selection bias based on the participants who chose to complete the survey. Additionally, this study occurred after protests for racial justice nationally and within Chicago and prior to the 2020 presidential election, which may have an influence COVID-19 VH, especially for non-Hispanic Black parents due to governmental mistrust [28].

However, this study's findings regarding parental COVID-19 vaccine hesitancy for children can aid in preparation for the widespread dissemination that is necessary for a successful vaccination program. Despite this study's limitations, this survey of parents identifies COVID-19 VH by demographic characteristics to help highlight communities that may be less amenable to receiving the COVID-19 vaccine for their child, once available. Additionally, this study synthesizes where parents receive information about the COVID-19 pandemic. The findings from this study and lessons learned from prior vaccine programs (e.g. annual influenza vaccine), including hesitancy and refusal, can help scientists, health care professionals and public health experts inform and promote a COVID-19 vaccine even before it becomes widely available in all age groups [11].

\section{Conclusion}

COVID-19 vaccine hesitancy may limit the successful dissemination and implementation of public health strategies to mitigate the global pandemic. Since children comprise approximately one-fifth of the U.S. population, it is essential to include children and their parents in efforts to achieve herd immunity and eventually disease eradication, which are the goals of every vaccination program. The highest rates of hesitancy were found in demographic groups that have been the most adversely affected by the pandemic, and this could potentially 
worsen gaps in disease burden, pushing us further from health equity. Efforts to disseminate information regarding the COVID-19 vaccine should be culturally tailored and delivered through effective sources to decrease the COVID-19 illness burden in disproportionately affected groups.

\section{Abbreviations}

CDC: Centers for Disease Control; Cl: Confidence Interval; OR: Odds Ratio: VH: Vaccine Hesitancy

\section{Acknowledgements}

Not applicable.

\section{Authors' contributions}

NA and JK conceptualized and designed the study, designed the data collection instruments, collected data, carried out the initial analyses, drafted the initial manuscript, and reviewed and revised the manuscript. NHG reviewed data analysis, made substantial contributions to the interpretation of the data, and critically reviewed the manuscript for important intellectual content. MD conceptualized and designed the study, supervised data analysis, and critically reviewed the manuscript for important intellectual content. LB helped with data collection, carried out the initial analyses, and critically reviewed the manuscript for important intellectual content. EG conceptualized and designed the study, designed the data collection instruments, helped with data collection, and critically reviewed the manuscript for important intellectual content. MM conceptualized and designed the study, reviewed and revised the data collection instruments, supervised and reviewed data analysis, and reviewed and revised the manuscript. All authors approved the final manuscript as submitted and agree to be accountable for all aspects of the work.

\section{Funding}

This project was funded by Stanley Manne Children's Research Institute COVID-19 Exploratory Springboard Award.

Dr. Heard-Garris's efforts were supported by the National Heart, Lung, and Blood Institute of the National Institutes of Health under award number K01HL147995. The content is solely the responsibility of the authors and does not necessarily represent the official views of the National Institutes of Health.

\section{Availability of data and materials}

The datasets used and/or analyzed during the current study are available from the corresponding author on reasonable request.

\section{Declarations}

\section{Ethics approval and consent to participate}

The study underwent an exempt determination request review by the Ann \& Robert H Lurie Children's Hospital of Chicago Institutional Review Board (IRB 2020-3715). All methods were performed in accordance with the relevant guidelines and regulations. The study was deemed exempt human subjects research based on category 2, human subjects research conducted through survey procedures. IRB waiver of documentation of informed consent was granted, meaning no signature needed to be obtained from study participants. Prior to initiating the survey, potential participants had the opportunity to review a study information sheet. The information sheet noted the voluntary nature of the research and that respondents indicated their consent to participate by providing answers to the survey questions. All survey participants were parents aged 18 years or older.

\section{Consent for publication}

Not applicable.

\section{Competing interests}

The authors have indicated they have no financial relationships relevant to this article to disclose.

\section{Author details}

'Division of Advanced General Pediatrics and Primary Care, Ann \& Robert H. Lurie Children's Hospital of Chicago, 225 E Chicago Ave, Box 162, Chicago, IL 60611, USA. ${ }^{2}$ Mary Ann \& J. Milburn Smith Child Health Outcomes, Research, and Evaluation Center; Stanley Manne Children's Research Institute, Ann \& Robert H. Lurie Children's Hospital of Chicago, Chicago, IL, USA. ${ }^{3}$ Department of Pediatrics, Northwestern University Feinberg School of Medicine, Chicago, IL, USA. ${ }^{4}$ Department of Medicine, Medical Social Sciences, and Preventive Medicine, Northwestern University Feinberg School of Medicine, Chicago, IL, USA. ${ }^{5}$ Division of Emergency Medicine, Ann \& Robert H. Lurie Children's Hospital of Chicago, Chicago, IL, USA.

Received: 11 February 2021 Accepted: 29 August 2021

Published online: 13 September 2021

\section{References}

1. Centers for Disease Control and Prevention (CDC). CDC COVID Data Tracker: Centers for Disease Control and Prevention; 2020. [updated 2020 Nov 23, cited 2021 July 6. Available from: https://covid.cdc.gov/covid-data-tracker/ \#cases_casesinlast7days

2. Dong E, Du H, Gardner L. An interactive web-based dashboard to track COVID-19 in real time. Lancet Infect Dis. 2020;20(5):533-4. https://doi.org/1 0.1016/S1473-3099(20)30120-1.

3. Illinois Department of Public Health (IDPH). COVID-19 Statistics. 2020 [cited 2021 July 6]. Available from: http://www.dph.illinois.gov/covid19/statistics.

4. chicago.gov. COVID Dashboard: Chicago COVID-19 Cases. 2021 [updated 2021 June 30, cited 2021 July 6]. Available from: https://www.chicago.gov/ city/en/sites/covid-19/home/covid-dashboard.html.

5. Zimmer C, Corum J, Wee SL. Coronavirus Vaccine Tracker nytimes.com: The New York Times; 2020. [updated 2020 Nov 24, cited 2021 Jul 6]. Available from: https://www.nytimes.com/interactive/2020/science/coronavirus-va ccine-tracker.html?name=styln-coronavirus-vaccines\&region=TOP_BA NNER\&block=storyline_menu_recirc\&action=click\&pgtype $=$ Article\&impression_id=73d1ac50-2b6e-11 eb-9a15-5767dafb2b73\&variant=1_ Show

6. Randolph HE, Barreiro LB. Herd immunity: understanding COVID-19. Immunity. 2020;52(5):737-41. https://doi.org/10.1016/j.immuni.2020.04.012.

7. The Annie E, Casey Foundation: Kids Count Data Center. Total population by child and adult populations in the United States. 2020 [cited 2021 Jul 6]. Available from: https://datacenter.kidscount.org/data/tables/99-total-popula tion-by-child-and-adult-populations\#detailed/1/any/false/1729,37,871 ,870,573,869,36,868,867,133/39,40,41/416,417.

8. Mandavilli A. Reaching 'Herd Immunity' is unlikely in the U.S., Experts Now Believe: New York Times; 2021. 2021;sect. The Coronvirus Outbreak

9. Quinn SC, Jamison A, An J, Freimuth VS, Hancock GR, Musa D. Breaking down the monolith: understanding flu vaccine uptake among African Americans. SSM Popul Health. 2018;4:25-36. https://doi.org/10.1016/j. ssmph.2017.11.003.

10. Jamison AM, Quinn SC, Freimuth VS. "You don't trust a government vaccine": narratives of institutional trust and influenza vaccination among African American and white adults. Soc Sci Med. 2019;221:87-94. https://doi. org/10.1016/j.socscimed.2018.12.020.

11. Kempe A, Saville AW, Albertin C, Zimet G, Breck A, Helmkamp L, et al. Parental hesitancy about routine childhood and influenza vaccinations: A national survey. Pediatrics. 2020;146(1):e20193852. https://doi.org/10.1542/ peds.2019-3852.

12. Hager ER, Quigg AM, Black MM, Coleman SM, Heeren T, Rose-Jacobs R, et al. Development and validity of a 2-item screen to identify families at risk for food insecurity. Pediatrics. 2010;126(1):e26-32. https://doi.org/10.1542/peds.2 009-3146.

13. Centers for Disease Control and Prevention (CDC). Community Assessment for Public Health Emergency Response (CASPER) Toolkit. 2nd ed. Atlanta: CDC; 2012.

14. Kazak A, Canter K, Phan-Vo T-L, McDonnell G, Hildenbrand A, Alderfer M, et al. COVID-19 exposure and family impact survey (CEFIS) 2020 [cited 2021 Jul 6]. Available from: https://www.healthcaretoolbox.org/tools-andresources/covid-19-cefis.html.

15. Karpman M, Zuckerman S, Gonzalez D. The well-being and basic needs survey. 2018 [cited 2021 Jul 6]. Available from: https://www.urban.org/sites/default/ files/publication/98919/the_well-being_and_basic_needs_survey_0.pdf. 
16. Zhang C, Conrad F. Speeding in Web Surveys: The tendency to answer very fast and its association with straightlining. SRM [Internet]. 2014 [cited 2021 July 6];8(2):127-35. Available from: https://ojs.ub.uni-konstanz.de/srm/article/ view/5453.

17. Centers for Disease Control and Prevention (CDC). Vaccinate with Confidence: Protect communities. Empower families. Stop myths. 2019 [cited 6 Jul 2021]. Available from: https://www.cdc.gov/vaccines/partners/va ccinate-with-confidence.html.

18. World Health Organization (WHO). Ten threats to global health in 2019. 2019 [cited 2021 July 6]. Available from: https://www.who.int/news-room/ spotlight/ten-threats-to-global-health-in-2019.

19. Jarrett C, Wilson R, O'Leary M, Eckersberger E, Larson HJ, Hesitancy SWGoV. Strategies for addressing vaccine hesitancy - a systematic review. Vaccine. 2015;33(34):4180-90. https://doi.org/10.1016/j.vaccine.2015.04.040.

20. Shui IM, Weintraub ES, Gust DA. Parents concerned about vaccine safety: differences in race/ethnicity and attitudes. Am J Prev Med. 2006;31(3):24451. https://doi.org/10.1016/j.amepre.2006.04.006.

21. Tai DBG, Shah A, Doubeni CA, Sia IG, Wieland ML. The disproportionate impact of COVID-19 on racial and ethnic minorities in the United States. Clin Infect Dis. 2020;72(4):703-6. https://doi.org/10.1093/cid/ciaa815.

22. Centers for Disease Control and Prevention (CDC). Health Equity Considerations and Racial and Ethnic Minority Groups cdc.gov 2020 [updated 2020 July 24, cited 2021 July 6]. Available from: https://www.cdc. gov/coronavirus/2019-ncov/community/health-equity/race-ethnicity. html\#fn1.

23. Centers for Disease Control and Prevention (CDC). Ensuring COVID-19 Vaccines Work cdc.gov 2020 [updated 2020 Dec 13, cited 2021 July 6]. Available from: https://www.cdc.gov/coronavirus/2019-ncov/vaccines/ effectiveness.html.

24. Quinn S, Jamison A, Musa D, Hilyard K, Freimuth V. Exploring the continuum of vaccine hesitancy between African American and white adults: results of a qualitative study. PLoS Curr. 2016;8. https://doi.org/10.1371/currents. outbreaks.3e4a5ea39d8620494e2a2c874a3c4201.

25. Santibanez TA, Nguyen KH, Greby SM, Fisher A, Scanlon P, Bhatt A, et al. Parental Vaccine Hesitancy and Childhood Influenza Vaccination. Pediatrics. 2020;146(6):e2020007609. https://doi.org/10.1542/peds.2020-007609.

26. Glanz JM, Wagner NM, Narwaney KJ, Kraus CR, Shoup JA, Xu S, et al. Webbased social media intervention to increase vaccine acceptance: a randomized controlled trial. Pediatrics. 2017:140(6):e20171117. https://doi. org/10.1542/peds.2017-1117.

27. Daley MF, Narwaney KJ, Shoup JA, Wagner NM, Glanz JM. Addressing Parents' vaccine concerns: a randomized trial of a social media intervention. Am J Prev Med. 2018;55(1):44-54. https://doi.org/10.1016/j.amepre.2018.04.010.

28. Richardson R. Black doctors endorse taking 'safe and effective' Covid-19 vaccine: NBC News; 2020. [cited 2021 July 6]. Available from: https://www. nbcnews.com/news/nbcblk/black-doctors-endorse-taking-safe-effectivecovid-vaccine-n1248637?cid=sm_npd_nn_tw_blk

\section{Publisher's Note}

Springer Nature remains neutral with regard to jurisdictional claims in published maps and institutional affiliations.

Ready to submit your research? Choose BMC and benefit from:

- fast, convenient online submission

- thorough peer review by experienced researchers in your field

- rapid publication on acceptance

- support for research data, including large and complex data types

- gold Open Access which fosters wider collaboration and increased citations

- maximum visibility for your research: over $100 \mathrm{M}$ website views per year

At $\mathrm{BMC}$, research is always in progress.

Learn more biomedcentral.com/submissions 